\title{
A GENERAL APPROACH TO BOUNDARY PROBLEMS ${ }^{1}$
}

\section{BY MARTIN SCHECHTER}

Communicated by Paul R. Garabedian, July 27, 1960

1. Introduction. In this paper we apply the method of negative norms to arbitrary boundary problems. The method has proved very powerful in dealing with elliptic problems (cf. [9]). Here we show that it is just as successful in general, without reference to the type of equation or boundary condition.

In the next section we define several negative norms and state two important representation theorems. Everything is done within the $L^{p}$ framework, $1<p<\infty$. In $\S 3$ we give necessary and sufficient conditions for certain boundary problems to have solutions. In $\$ 4$ it is noted that these theorems give new results even in the case of elliptic problems. In $\$ 5$ it is shown how our methods can be employed to give complete aswers for the Višik-Sobolev problems.

Proofs of all our theorems will appear elsewhere.

2. Negative norms. Let $G$ be a bounded domain in Euclidean $n$-space $E^{n}$ with boundary $\partial G$ of $\operatorname{class}^{2} C^{\infty}$. Let $C^{\infty}(\bar{G})$ denote the set of all complex valued functions infinitely differentiable on $\bar{G}$, the closure of $G$. If $\mu=\left(\mu_{1}, \mu_{2}, \cdots, \mu_{n}\right)$ is any multi-index of length $|\mu|=\mu_{1}+\mu_{2}+\cdots+\mu_{n}$, we set

$$
D^{\mu}=\partial^{|\mu|} / \partial x_{1}^{\mu_{1}} \partial x_{2}^{\mu_{2}} \cdots \partial x_{n}^{\mu_{n}} .
$$

If $s$ is any non-negative integer and $p$ is any real number greater than one, we define

$$
\begin{aligned}
& \|u\|_{s, p}=\left(\int_{G} \sum_{|\mu| \leq s}\left|D^{\mu} u\right|^{p} d x\right)^{1 / p}, \\
& \|u\|_{-s, p}=\operatorname{lidu.b.}_{v \in C^{\infty}(\bar{G})} \frac{|(u, v)|}{\|v\|_{s, p^{\prime}}}, \quad p^{\prime}=\frac{p}{p-1}
\end{aligned}
$$

for functions $u \in C^{\infty}(\bar{G})$, where $(u, v)=\int_{G} u \bar{v} d x$. Denote the completions of $C^{\infty}(\bar{G})$ with respect to these norms by $H^{s, p}(G)$ and $H^{-s, p}(G)$, respectively. They are obviously Banach spaces. Concerning them we state

1 The work presented in this paper was supported by the Institute of Mathematical Sciences, New York University, under Contract AT(30-1)-480 with the U. S. Atomic Energy Commission.

${ }^{2}$ Some assumptions are made for convenience only. Our results hold under less restrictive hypotheses. 
TheOREM 2.1. For every bounded linear functional $F(w)$ on $H^{s, p}(G)$ there is an $f \in H^{-s, p^{\prime}}(G)$ such that $F(w)=(w, f)$ for all $w \in H^{s, p}(G)$. Conversely, every bounded linear functional $G(u)$ on $H^{-s, p}(G)$ can be represented by $(u, g)$, where $g \in H^{s, p^{\prime}}(G)$.

Next, let $C_{0}^{\infty}(G)$ denote the set of all $u \in C^{\infty}(\bar{G})$ which vanish near $\partial G$. Assume that there is given a linear space $V$ of functions such that $C_{0}^{\infty}(G) \subseteq V \subseteq C^{\infty}(\bar{G})$. Corresponding to $V$ we can define the norm

$$
|u|_{-s, p}=\operatorname{liu.b.}_{v \in V} \frac{|(u, v)|}{\|v\|_{s, p^{\prime}}} .
$$

Complete $C^{\infty}(\bar{G})$ with respect to this norm, and call the resulting Banach space $\widetilde{H}^{-s, p}(G)$.

Theorem 2.2. Let $V^{s, p}(G)$ denote the closure of $V$ in $H^{s, p}(G)$. Then every bounded linear functional $F(w)$ on $V^{s, p}(G)$ can be represented in the form $F(w)=(w, f)$, where $f \in H^{-s, p^{\prime}}(G)$. Conversely, every bounded linear functional $G(u)$ on $\widetilde{H}^{-s, p}(G)$ equals $(u, g)$ for some $g \in V^{s, p^{\prime}}(G)$.

3. Boundary problems. Assume that there is also given a partial differential operator of order $m \geqq 1$

$$
A=\sum_{|\mu| \leq m} a_{\mu}(x) D^{\mu}
$$

with coefficients ${ }^{3} a_{\mu}(x)$ in $C^{\infty}(\bar{G})$. The formal adjoint $A^{\prime}$ of $A$ is defined by

$$
A^{\prime} u=\sum_{|\mu| \geqq m}(-1)^{|\mu|} D^{\mu}\left(\bar{a}_{\mu} u\right)
$$

and it is easily checked that

$$
(A u, v)=\left(u, A^{\prime} v\right)
$$

whenever one of the functions $u, v$ is in $C_{0}^{\infty}(G)$. We define $V^{\prime}$ as the set of all those $v \in C^{\infty}(\bar{G})$ which satisfy (3.1) whenever $u \in V$. Clearly, $C_{0}^{\infty}(G) \subseteq V^{\prime}$. Corresponding to (2.2) we set

$$
|u|_{-s, p}^{\prime}=\operatorname{liu.b}_{v \in V^{\prime}} \frac{|(u, v)|}{\|v\|_{s, p^{\prime}}}
$$

and denote the completion of $C^{\infty}(\bar{G})$ with respect to this norm by $\tilde{H}^{\prime-s, p}(G)$. The closure of $V^{\prime}$ in $H^{s, p}(G)$ is denoted by $V^{\prime s, p}(G)$.

By (2.1), (2.2), and (3.2) we have

${ }^{8}$ Cf. footnote 2. 


$$
\begin{aligned}
& |(u, v)| \leqq\|u\|_{-s, p}\|v\|_{s, p^{\prime}}, \\
& |(u, v)| \leqq|u|_{-s, p}\|v\|_{s, p^{\prime}}, \\
& |(u, v)| \leqq|u|_{-s, p}^{\prime}\|v\|_{s, p^{\prime}},
\end{aligned}
$$

$$
\begin{array}{r}
u \in C^{\infty}(\bar{G}), v \in C^{\infty}(\bar{G}), \\
u \in C^{\infty}(\bar{G}), v \in V, \\
u \in C^{\infty}(\bar{G}), v \in V^{\prime} .
\end{array}
$$

By completion, we have that (3.3) holds for $u \in H^{-s, p}(G)$, $v \in H^{s, p}(G)$, (3.4) holds for $u \in \tilde{H}^{-s, p}(G), v \in V^{s, p}(G)$, and (3.5) holds for $u \in \tilde{H}^{\prime-s, p}(G), v \in V^{\prime s, p}(G)$.

Let $N$ (resp. $N^{\prime}$ ) be the set of those $u \in V$ (resp. $v \in V^{\prime}$ ) satisfying $A u=0$ (resp. $A^{\prime} v=0$ ) in $G$. We shall assume that both $N$ and $N^{\prime}$ are finite dimensional. ${ }^{4}$ If $L$ is any space of functions containing $N$ (resp. $N^{\prime}$ ) we let $L / N$ (resp. $L / N^{\prime}$ ) denote the set of all $u \in L$ satisfying $(u, v)=0$ for all $v \in N$ (resp. $\left.v \in N^{\prime}\right)$. The letter $s$ will denote an arbitrary non-negative integer, $t, r$, arbitrary integers, and $p, q$, real numbers greater than one, with $p^{\prime}=p /(p-1), q^{\prime}=q /(q-1)$. When $t$ is positive, we set $\widetilde{H}^{t, p}(G)=\tilde{H}^{t, p}(G)=H^{t, p}(G)$. We write $u \in H^{-\infty}(G)$ (resp. $\widetilde{H}^{-\infty}(G), \widetilde{H}^{\prime-\infty}(G)$ ) when $u \in H^{t, q}(G)$ (resp. $\widetilde{H}^{t, q}(G), \widetilde{H}^{\prime t, q}(G)$ ) for some $t$ and $q$.

Theorem 3.1. Let $f \in \widetilde{H}^{\prime-\infty}(G)$ be given. Then there exists $a u \in H^{r, p}(G)$ such that

$$
\left(u, A^{\prime} v\right)=(f, v) \quad \text { for all } v \in V^{\prime}
$$

if, and only if,

$$
|(f, v)| \leqq c\left\|A^{\prime} v\right\|_{-r, p^{\prime}} \quad \text { for all } v \in V^{\prime} .
$$

There exists a $u \in V^{s, p}(G)$ satisfying (3.6), if, and only if,

$$
|(f, v)| \leqq c\left|A^{\prime} v\right|_{-8, p^{\prime}} \quad \text { for all } v \in V^{\prime} .
$$

Corollary 3.1. A necessary and sufficient condition that for each $f \in \tilde{H}^{\prime t, q}(G) / N^{\prime}$ there exist a $u \in H^{r, p}(G)$ satisfying (3.6) is that

$$
\|v\|_{-t, q^{\prime}} \leqq c\left\|A^{\prime} v\right\|_{-r, p^{\prime}} \quad \text { for all } v \in V^{\prime} / N^{\prime} .
$$

$A$ necessary and sufficient condition that $a u \in V^{s, p}(G)$ satisfy (3.6) for each such $f$ is that

$$
\|v\|_{-t, q^{\prime}} \leqq c\left|A^{\prime} v\right|_{-s, p^{\prime}} \quad \text { for all } v \in V^{\prime} / N^{\prime} .
$$

CoROLlary 3.2. The following statements are equivalent.

(a) For every $f \in H^{s, p}(G) / N^{\prime}$ there is a $u \in V^{m+s, p}(G)$ and a sequence $\left\{u_{k}\right\} \in V$ such that $\left\|u_{k}-u\right\|_{m+s, p} \rightarrow 0,\left\|A u_{k}-f\right\|_{s, p} \rightarrow 0$ as $k \rightarrow \infty$.

(b) For every $f \in H^{s, p}(G) / N^{\prime}$ there is a $u \in V^{m+s, p}(G)$ satisfying (3.6).

(c) $\|v\|_{-s, p^{\prime}} \leqq c\left|A^{\prime} v\right|_{-m-s, p^{\prime}}$ for all $v \in V^{\prime} / N^{\prime}$.

4 Infinite dimensional $N$ and unbounded $G$ will be considered elsewhere. 
Corollary 3.3. Given $f \in \tilde{H}^{\prime-\infty}(G)$. The following statements are equivalent.

(a) There is a $u \in H^{-s, p}(G)$ satisfying (3.6).

(b) $f \in \tilde{H}^{\prime-m-s, p}(G) / N^{\prime}$ and there is a $u \in H^{-s, p}(G)$ and a sequence $u_{k} \in V$ such that $\left\|u_{k}-u\right\|_{-s, p} \rightarrow 0,\left|A u_{k}-f\right|_{-m-s, p}^{\prime} \rightarrow 0$ as $k \rightarrow \infty$.

(c) $|(f, v)| \leqq c\left\|A^{\prime} v\right\|_{s, p^{\prime}}$ for all $v \in V^{\prime}$.

Corollary 3.4. For each $f \in \tilde{H}^{\prime-m-s, p}(G) / N^{\prime}$ there is a $u \in H^{-s, p}(G)$ satisfying (a) or (b) of Corollary 3.3, if, and only if,

$$
\|v\|_{m+s, p^{\prime}} \leqq c\left\|A^{\prime} v\right\|_{s, p^{\prime}} \quad \text { for all } v \in V^{\prime} / N^{\prime} .
$$

4. Elliptic problems. We shall say that $A$ is elliptic on $V$ if

(a) $N$ and $N^{\prime}$ are finite dimensional.

(b) For every $s \geqq 0$ and $p>1$ there is a constant $c_{s, p}$ such that

$$
\begin{array}{ll}
\|u\|_{m+s, p} \leqq c_{s, p}\|A u\|_{s, p} & \text { for } u \in V / N, \\
\|v\|_{m+s, p} \leqq c_{s, p}\left\|A^{\prime} v\right\|_{s, p} & \text { for } v \in V^{\prime} / N^{\prime} .
\end{array}
$$

(c) For every $f \in C^{\infty}(\bar{G}) / N^{\prime}$ there is a $u \in V$ such that $A u=f$; for every $g \in C^{\infty}(\bar{G}) / N$ there is a $v \in V^{\prime}$ such that $A^{\prime} v=g$.

It has been shown that when $A$ is a properly elliptic operator, these conditions are satisfied for a wide variety of spaces $V$ (cf. $[2 ; 3 ; 8])$. Here we shall be concerned with conclusions which can be drawn from (a)-(c) without further assumptions.

Theorem 4.1. If $A$ is elliptic on $V$, then

$$
\begin{aligned}
\|u\|_{-s, p} & \leqq c_{s, p}|A u|_{-m-s, p}^{\prime} \quad \text { for } u \in V / N, \\
\|v\|_{-s, p} \leqq c_{s, p}\left|A^{\prime} v\right|_{-m-s, p} & \text { for } v \in V^{\prime} / N^{\prime} .
\end{aligned}
$$

THEOREM 4.2. For each $f \in \tilde{H}^{\prime-m-s, p}(G) / N^{\prime}$ there is a $u \in H^{-s, p}(G)$ and a sequence $\left\{u_{k}\right\} \in V$ such that $\left\|u_{k}-u\right\|_{-s, p} \rightarrow 0$ and $\left|A u_{k}-f\right|_{-m-s, p}^{\prime}$ $\rightarrow 0$ as $k \rightarrow \infty$.

Theorem 4.3. If $u \in H^{-\infty}(G)$ and $\left|\left(u, A^{\prime} v\right)\right| \leqq c\|v\|_{m+s, p^{\prime}}$ for all

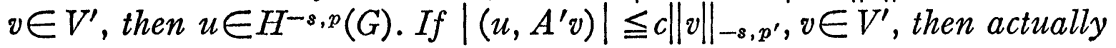
$u \in V^{m+s, p}(G)$.

5. Višik-Sobolev problems. Let $C_{0}^{\infty}\left(E^{n}\right)$ be the space of infinitely differentiable functions with compact support in $E^{n}$ and let $H^{s, p}\left(E^{n}\right)$ be the closure of $C_{0}^{\infty}\left(E^{n}\right)$ with respect to the norm

$$
\|\phi\|_{s, p}^{E^{n}}=\left(\int_{E n} \sum_{|\mu| \leqq s}\left|D^{\mu} \phi\right|^{p} d x\right)^{1 / p}
$$

We can introduce a negative norm on $E^{n}$ as follows: Set 


$$
\|\phi\|_{-s, p}^{E^{n}}=\underset{\psi \in C_{0}^{\infty}\left(E^{n}\right)}{\operatorname{lub} .} \frac{|\langle\phi, \psi\rangle|}{\|\psi\|_{8, p^{\prime}}},
$$

where $\langle\phi, \psi\rangle=\int_{E^{n}} \phi \bar{\psi} d x$. Denote the completion of $C_{0}^{\infty}\left(E^{n}\right)$ with respect to \|\|$_{-s, p}^{n}$ by $H^{-s, p}\left(E^{n}\right)$. This space may be defined in other ways (cf. $[4 ; 6]$ ).

For any $\phi \in C_{0}^{\infty}\left(E^{n}\right)$, let $\phi_{G}$ be the restriction of $\phi$ to $G$. An element $u \in H^{-s, p}\left(E^{n}\right)$ is said to be in $H_{\bar{G}}^{-s, p}$ if $\langle u, \phi\rangle=0$ for all $\phi \in C_{0}^{\infty}(E)$ such that $\phi_{G}=0$. Such functions (distributions) $u$ are said to have their support contained in $\bar{G}$. It can be shown that $H_{\bar{G}}^{-s, p}$ is isomorphic to $H^{-8, p}(G)$.

We set $M^{-s, p}$ equal to the space of all $h \in H^{-s, p}\left(E^{n}\right)$ which satisfy $\langle h, \phi\rangle=0$ whenever $\phi \in C_{0}^{\infty}\left(E^{n}\right)$ and $\phi_{G} \in V^{\prime}$. Clearly $M^{-8, p}$ is a subspace of $H_{\bar{G}}^{-s, p}$. Now assume that the coefficients ${ }^{5}$ of $A$ are in $C_{0}^{\infty}\left(E^{n}\right)$. For every $h \in H^{-s, p}\left(E^{n}\right)$ there is an element $g \in H^{-m-s, p}\left(E^{n}\right)$ such that

$$
\left\langle h, A^{\prime} \phi\right\rangle=\langle g, \phi\rangle
$$

for all $\phi \in C_{0}^{\infty}\left(E^{n}\right)$. We define $A h$ to be $g$. Now suppose $f \in H_{\bar{G}}^{-m-s, p}$ and $u \in H_{\bar{G}}^{-s, p}$ satisfy

$$
\left\langle u, A^{\prime} \phi\right\rangle=\langle f, \phi\rangle
$$

for all $\phi \in C_{0}^{\infty}\left(E^{n}\right)$ such that $\phi_{G} \in V^{\prime}$. Then by (5.1) $\langle A u-f, \phi\rangle=0$ for all such $\phi$. Hence $A u-f \in M^{-m-s, p}$. Conversely, if $A u-f \in M^{-m-s, p}$, then (5.2) holds. Thus, given $f \in H_{\bar{G}}^{-m-s, p}$, the problem of finding a $u \in H_{\bar{G}}^{-s, p}$ such that (5.2) holds when $\phi \in C_{0}^{\infty}\left(E^{n}\right)$ and $\phi_{G} \in V^{\prime}$ is equivalent to finding a $u \in H_{\bar{G}}^{-s, p}$ such that $A u-f \in M^{-m-s, p}$. Following Lions we call this a Višik-Sobolev problem. For previous results on such problems we refer to Lions $[4 ; 5]$ and Višik-Sobolev [10].

THEOREM 5.1. Given $f \in H_{\bar{G}}^{-m-s, p}$, there is a $u \in \bar{H}_{\bar{G}}^{-s, p}$ such that $A u-f \in M^{-m-s, p}$ if, and only if,

$$
|\langle f, \phi\rangle| \leqq c\left\|A^{\prime} \phi\right\|_{s, p^{\prime}}^{G}
$$

for all $\phi \in C_{0}^{\infty}\left(E^{n}\right)$ such that $\phi_{G} \in V^{\prime}$.

ThEOREM 5.2. For every $f \in H_{\bar{G}}^{-m-s, p} / N^{\prime}$ there is a $u \in H_{\bar{G}}^{-s, p}$ such that $A u-f \in M_{\bar{G}}^{-m-s, p}$ if, and only if,

$$
\|v\|_{m+s, p^{\prime}}^{G} \leqq c\left\|A^{\prime} v\right\|_{s, p^{\prime}}^{G} \quad \text { for all } v \in V^{\prime} .
$$

Corollary 5.1. If $A$ is elliptic on $V$, then the Visik-Sobolev problem considered has a solution for each $f \in H_{\bar{G}}^{-m-s, p} / N^{\prime}$.

${ }^{5} \mathrm{Cf}$. footnote 2.

${ }^{6}$ By this we mean that $\langle f, \phi\rangle=0$ for all $\phi \in C_{0}^{\infty}\left(E^{n}\right)$ such that $\phi_{G} \in N^{\prime}$. 


\section{REFERENCES}

1. Shmuel Agmon, The $L_{p}$ approach to the Dirichlet problem. I, Technical Note No. 7, The Hebrew University, Jerusalem, 1959.

2. Shmuel Agmon, Avron Douglis, and Louis Nirenberg, Estimates near the boundary for solutions of elliptic partial differential equations satisfying general boundary conditions. I, Comm. Pure Appl. Math. vol. 12 (1959) pp. 623-727.

3. F. E. Browder, Estimates and existence theorems for elliptic boundary value problems, Proc. Nat. Acad. Sci. U.S.A., vol. 45 (1959) pp. 365-372.

4. J. L. Lions, Conditions aux limites de Višik-Soboleff et problèmes mixtes, C. R. Acad. Sci. Paris vol. 244 (1957) pp. 1126-1128.

5. - Lectures on elliptic partial differential equations, Tata Institute of Fundamental Research, Bombay, 1957.

6. Enrico Magenes and Guido Stampacchia, I problemi al contorno per le equazioni differenziali di tipo ellittico, Ann. Scuola Norm. Sup. Pisa, Serie 3, vol. 12 (1958) pp. 247-358.

7. Martin Schechter, General boundary value problems for elliptic partial differential equations, Comm. Pure Appl. Math. vol. 12 (1959) pp. 457-486.

8. - Remarks on elliptic boundary value problems, Comm. Pure Appl. Math. vol. 12 (1959) pp. 561-578.

9. - Negative norms and boundary problems, Ann. of Math. vol. 72 (1960) (No. 3) to appear.

10. M. I. Višik and S. L. Sobolev, General formulation of certain boundary problems for elliptic partial differential equations, Dokl. Akad. Nauk SSSR (N.S.) vol. 111 (1956) pp. 521-523. (Russian).

Institute of Mathematical Sciences, New YoRk University 